\title{
MiR-125b-5p enclosed in hypoxic HK2 cell-derived extracellular vesicles alleviates renal ischemia- reperfusion injury by regulating NLRC5
}

\section{Type}

Research paper

\section{Keywords}

Hypoxia, miR-125b-5p, NLRC5, renal ischemia-reperfusion(I/R) injury, extracellular vesicles(EVs)

\begin{abstract}
Introduction

In this study, we validated the changes in microRNA (miRNA) expression in hypoxic human kidney 2 (HK2) cell-derived extracellular vesicles (EVs). Additionally, we investigated the mechanism by which miRNA that are derived from EVs alleviated renal ischemia-reperfusion (I/R) injury.
\end{abstract}

\section{Material and methods}

HK2 cells were treated in a hypoxic chamber $(1 \%$ O2) for $12 \mathrm{~h}$.EVs were obtained as supernatant from ultracentrifugation and characterized. After examining 16 differentially expressed EV-miRNAs between normoxic and hypoxic conditions by RT-PCR and bioinformatics analysis, miR-125b-5p was selected for further analysis. Normoxic and hypoxic HK2 cells-derived EVs as well as EVs that were isolated from miR-125b-5p negative control (miR-NC)-transfected or miR-125b-5p mimic (miR$\mathrm{MI}$ )-transfected HK2 cells were injected in mice with renal I/R injury. The degree of renal injury was assessed by periodic acid-Schiff staining, renal tubule injury score, and plasma creatinine levels. Bioinformatics analysis was performed to determine the potential target genes of differentially expressed miRNAs. RT-PCR, western blotting, luciferase reporter assay, and immunohistochemistry were performed to investigate the relationship between miR-125b-5p and the NLR family CARD domain containing 5 (NLRC5).

\section{Results}

RT-PCR revealed that from 16 differentially expressed miRNAs, four EV-miRNAs were upregulated. Animal study showed that miR-125b-5p overexpression in EVs alleviated renal I/R injury. Bioinformatics analysis predicted that NLRC 5 was targeted by miR-125b-5p. Moreover, the relationship between miR-125b-5p and NLRC5 was also validated.

\section{Conclusions}

There were several miRNAs that were upregulated (including miR-125b-5p) in hypoxic HK2 cells. Hypoxia induced EVs that alleviate renal IRI, can be attributed to miR-125b-5p for targeting NLRC5. 
MiR-125b-5p enclosed in hypoxic HK2 cell-derived extracellular vesicles alleviates renal ischemia-reperfusion injury by regulating NLRC5

Kai Xu ${ }^{1,2,3^{*}}$, Lifeng Zhang ${ }^{3 *}$, Lei Zhang ${ }^{3 *}$, Lei Zhang ${ }^{1,2^{*}}$, Ze Zhang ${ }^{3 *}$, Siwei Qian ${ }^{1,2}$, Xiangyu Zou $^{4}$, Shuqiu Chen ${ }^{1,2}$, Li Zuo ${ }^{3 \#}$, Ming Chen ${ }^{1,2 \#}$, Guangyuan Zhang ${ }^{1,2 \#,}$

${ }^{1}$ Department of Urology, Zhongda Hospital, Southeast University, Nanjing, Jiangsu 210009, P.R. China;

${ }^{2}$ Institute of Urology, Surgical Research Center, School of Medicine, Southeast University, Nanjing, Jiangsu 210009, P.R. China;

${ }^{3}$ Department of Urology, The Affiliated Changzhou No. 2 People's Hospital of Nanjing Medical University, Changzhou, China;

${ }^{4}$ Department of Urology, Shanghai Children's Medical Center, Shanghai Jiaotong University School of Medicine, Shanghai 200127, P.R. China

*Equal contributors

\#Correspondence to: Guangyuan Zhang or Ming Chen, or Li Zuo

Department of Urology, Zhongda Hospital, Southeast University, 87 Dingjiaqiao, Gulou, Nanjing, Jiangsu 210009, P.R. China

E-mail: zgy0879@qq.com (G.Zhang),zz261608@yeah.net (M.Chen), zuoli1978@hotmail.com (L. Zuo) 


\begin{abstract}
Introduction In this study, we validated the changes in microRNA (miRNA) expression in hypoxic human kidney 2 (HK2) cell-derived extracellular vesicles (EVs). Additionally, we investigated the mechanism by which miRNA that are derived from EVs alleviated renal ischemia-reperfusion (I/R) injury.
\end{abstract}

Methods HK2 cells were treated in a hypoxic chamber $\left(1 \% \mathrm{O}_{2}\right)$ for $12 \mathrm{~h} . \mathrm{EVs}$ were obtained as supernatant from ultracentrifugation and characterized. After examining 16 differentially expressed EV-miRNAs between normoxic and hypoxic conditions by RT-PCR and bioinformatics analysis, miR-125b-5p was selected for further analysis. Normoxic and hypoxic HK2 cells-derived EVs as well as EVs that were isolated from miR-125b-5p negative control (miR-NC)-transfected or miR-125b-5p mimic (miR-MI)-transfected HK2 cells were injected in mice with renal I/R injury. The degree of renal injury was assessed by periodic acid-Schiff staining, renal tubule injury score, and plasma creatinine levels. Bioinformatics analysis was performed to determine the potential target genes of differentially expressed miRNAs. RT-PCR, western blotting, luciferase reporter assay, and immunohistochemistry were performed to investigate the relationship between miR-125b-5p and the NLR family CARD domain containing 5 (NLRC5).

Resullts RT-PCR revealed that from 16 differentially expressed miRNAs, four EV-miRNAs were upregulated. Animal study showed that miR-125b-5p overexpression in EVs alleviated renal I/R injury. Bioinformatics analysis predicted that NLRC5 was targeted by miR-125b-5p. Moreover, the relationship between miR-125b-5p and NLRC5 was also validated.

Conclusion There were several miRNAs that were upregulated (including miR-125b-5p) in hypoxic HK2 cells. Hypoxia induced EVs that alleviate renal IRI, can be attributed to miR$125 b-5 p$ for targeting NLRC5.

Keywords: miR-125b-5p, NLRC5, renal ischemia-reperfusion(I/R) injury, hypoxia, extracellular vesicles(EVs) 


\section{Introduction}

Renal ischemia-reperfusion(I/R) injury is the leading cause of acute kidney injury (AKI), which is one of the most common and serious complications of kidney transplantation and major surgery. I/R injury is associated with increased morbidity and mortality, and often, patients with renal I/R injury have a poor prognosis[1-4]. Therefore, an effective therapeutic strategy needs to be developed to treat renal I/R injury.

Remote ischemic preconditioning (RIPC) protects not only local but also remote organs[5]. RIPC represents an effective strategy for attenuating organ injury and has been successfully performed to protect the kidneys, heart, and other organs from I/R injury. Recently, as novel circulating mediators, extracellular vesicles $(\mathrm{EVs})$ have been proposed to mediate the protective effects of RIPC[6-9]. In our previous study, we proved that EVs mediated the protection effect of RIPC in renal I/R injury; however, the specific protective mechanisms involved in the process are unknown[10].

EVs exert their biological functions by transmitting their cargos into recipient cells[11]. EVs have been reported to comprise large amount of proteins, lipids, and nucleic acids that can regulate various physiological or pathological functions, such as blood vessel growth, tumor cell invasion and metastasis, and immune responses[12]. RNA is considered to be the main bioactive cargo of EVs, and microRNAs (miRNAs) are reported to be enriched in EVs[1316]. Recently, the significance of miRNAs in I/R injury has gradually emerged. MiRNAs are small, endogenous RNAs, which are approximately 20-24 nucleotides in length and have been shown to play important roles in I/R injury[17-19]. Some miRNAs, such as miR-21[20] and miR-489[21], have been reported to be beneficial in reducing I/R injury.

In our previous study, we performed high-throughput sequencing of EV-miRNAs, which were obtained from hypoxic-treated and normoxic-treated human kidney (HK2) cells. Compared to EVs under normoxic conditions, 16 EV-packed miRNAs (EV-miRNAs) under hypoxia found to be were substantially upregulated[22]. However, the mechanisms underlying the functioning of these miRNAs as well as their downstream mechanisms, remain unclear. 
In this study, expression of 16 EV-miRNAs was verified by RT-PCR. Subsequently, miRNAs with protective effects against renal $\mathrm{I} / \mathrm{R}$ injury were selected by bioinformatics analysis. Furthermore, the protective effects of HK2 cell-derived EVs under hypoxia in renal I/R injury, and related mechanisms were then verified by in vitro and in vivo experiments.

\section{Materials and Methods}

\section{Animal experiments}

All animal experiments were performed using male C57BL/6 mice (weight, 20-25 g; age, 10-12 weeks), which were purchased from the Comparative Medicine Center of Yangzhou University (Yangzhou, China).

Mice were nurtured by the Animal Experimental Center of Southeast University Medical College (SPF feed, unlimited water, $12 \mathrm{~h}$ of alternate lighting, temperature $24 \pm 2{ }^{\circ} \mathrm{C}$, humidity 40\%-60\%). Animal experiments were performed in accordance with the regulations of the National Animal Experiment Management, Jiangsu Province Animal Experiment Management, and the Southeast University Animal Center Management. All protocols were approved by Committee on the Ethics of Animal Experiments of Southeast University (Nanjing, China). Surgeries were performed using intraperitoneal sodium pentobarbital anesthesia, and all efforts were made to minimize animal suffering.

To induce a bilateral renal I/R injury model in mice, we subjected bilateral kidneys to ischemia for $35 \mathrm{~min}$. To treat renal I/R injury, EVs were intravenously injected in mice $24 \mathrm{~h}$ before operating for I/R. Mice were sacrificed $24 \mathrm{~h}$ following reperfusion, and their kidneys and blood were collected for examination. Collectively, rats were divided into 4 groups: Sham group (n=6); IRI+PBS group (n=6); IRI+HPY-EVs; IRI+NOR-EVs group $(n=6)$.

\section{Cell culture and transfection}

HK2 cells were obtained from a commercial source (Institutes for Biological Sciences, Shanghai, China) and cultured in the Dulbecco's modified Eagle medium/F-12 Nutrient Mixture (DMEM/F12, Thermo Fisher, USA), which was supplemented with $10 \%$ fetal bovine serum (FBS, ExCell Bio, China), $50 \mu \mathrm{g} / \mathrm{ml}$ streptomycin, and $50 \mu \mathrm{g} / \mathrm{ml}$ penicillin (Gibco, 
USA) at $37^{\circ} \mathrm{C}$ in a humidified atmosphere with $5 \% \mathrm{CO}_{2}$. Culture medium was changed once or twice a week (according to cell growth). HK2 cells were divided into two groups: negative control group and mimic group. The miR-125b-5 negative control (miR-NC) and miR-125b5p mimics (miR-MI) (Ribobio, Guangzhou, China) were transfected using Lipofectamine 2000 (Invitrogen; Thermo Fisher). Briefly, a day before transfection, HK2 cells were seeded in a $10-\mathrm{cm}$ dish at $70 \%$ confluence. Nucleotides formed transfection complexes with Lipofectamine 2000 and then, added to HK2 cells, which were then incubated for $6-8 \mathrm{~h}$ before replacing the antibiotic-free medium.

\section{Hypoxic exposure}

Hypoxic exposure was accomplished using a hypoxic chamber at $37^{\circ} \mathrm{C}$ with $1 \% \mathrm{O}_{2}$ and $5 \%$ $\mathrm{CO}_{2}$ in a humidified environment, which was balanced with nitrogen. The oxygen tension in the chamber was monitored and maintained using computerized sensor probe.

\section{EVs isolation}

In brief, HK2 cells were cultured in a FBS-deprived medium for 2 days. Condition medium was carefully collected and subjected to a series of centrifugation at $500 \times \mathrm{g}$ for $5 \mathrm{~min}$ to remove the HK2 cell components and at 2,000 $\times \mathrm{g}$ for $20 \mathrm{~min}$ to remove the debris. Resultant supernatant was subjected to ultracentrifugation in a SW41 swing rotor (Beckman Coulter, Fullerton, CA, USA) at $100,000 \times \mathrm{g}$ for $2 \mathrm{~h}$ at $4{ }^{\circ} \mathrm{C}$. Pellets were resuspended, washed with 1 $\mathrm{mL}$ of cold PBS, and again ultracentrifugated $\left(100,000 \times \mathrm{g}, 2 \mathrm{~h}, 4{ }^{\circ} \mathrm{C}\right)$. Prolonged ultracentrifugation time did not damage EVs. Finally, pellets were resuspended in cold PBS and immediately stored at $-80{ }^{\circ} \mathrm{C}$.

\section{Transmission electron microscopy (TEM)}

TEM was performed by Electron Microscopy Core of Southeast University. Briefiy, $10 \mu \mathrm{L}$ of the EV pellet solution was loaded onto formvar/carbon-coated 200-mesh copper electron microscopy grid, incubated at room temperature for $5 \mathrm{~min}$, and then subjected to negative staining with $3 \%$ aqueous phosphor-tungstic acid for $1 \mathrm{~min}$. The grid was washed with particle-free PBS and allowed to semidry at room temperature before observing under TEM (Tokyo, Japan).

\section{Western blot analysis}

Cell lysates and EV proteins were extracted using chilled RIPA buffer. Protein concentration 
was quantified using the BCA Protein Assay Kit (Keygen Biotech, Nanjing, China). Proteins were separated by $10 \%$ sodium dodecyl sulfate-polyacrylamide gel electrophoresis and transferred onto a polyvinylidene difluoride membrane (Millipore, MA, USA). Membrane was blocked with $5 \%$ bovine serum albumin (BSA) for $1 \mathrm{~h}$ at room temperature and then immunoblotted overnight with primary antibodies at $4{ }^{\circ} \mathrm{C}$. Further, membrane was washed three times and incubated with the secondary antibodies at $37^{\circ} \mathrm{C}$ for $1 \mathrm{~h}$. Signal was developed using enhanced chemiluminescence (ECL, Beyotime, Shanghai, China). Following primary antibodies were used: polyclonal rabbit anti-human/mouse NLRC5 (1:500, LSB5328, LifeSpan), polyclonal rabbit anti-human HSP70 (1:10,000, ab79852, Abcam), monoclonal rabbit anti-human CD81 (1:500, ab109201, Abcam), polyclonal rabbit antihuman CD63 (1:10,000, ab216130, Abcam), and polyclonal rabbit anti-human GAPDH (1:3,000, 10494-1-AP, Proteintech). Horseradish peroxidase (HRP)-conjugated Affinipure Goat anti-rabbit IgG $(\mathrm{H}+\mathrm{L})(1: 10,000, \mathrm{SA} 00001-2$, Proteintech) was used as secondary antibody.

\section{RNA extraction and real-time PCR}

Total RNA was isolated using the RNease Isolation Kit (Qiagen, Valencia, CA, USA) according to the manufacturer's instructions. RNA concentrations were detected using the NanoDrop 2000 spectrophotometer (Thermo Fisher Scientific). We used the ViiA 7 Real-time PCR System (Applied Biosystem, Shanghai, China) for quantitative detection of miR-125b5p. Stem-loop-like miRNA-specific reverse transcription primers can specifically transcribe mature miRNAs. U6 was used as an internal control. NLRC5 mRNA expression was measured by one-step RT-PCR. GAPDH was used as an internal control. The following primers were used to perform RT-PCR: NLRC5, forward: 5'-

CAGATGGTGGAAACTTTTAGCC-3' and reverse: 5'-AACTTCCTTAGCACCTGGATCA3'; MIR-125b-5p forward: 5'-GCTCCCTGAGACCCTAAC-3' and reverse: 5'GTGCGTGTCGTGGAGTCG-3'; U6 forward: 5'-GCTTCGGCAGCACATATACTAAAAT-3' and reverse: 5'-CGCTTCACGAATTTGCGTGTCAT-3'; GAPDH forward: 5'-AGA ACA TCA TCC CTG CAT CC--3' and reverse 5'-ACA CAT TGG GGG TAG GAA CA--3'. We analyzed the relative quantity of RNA by using the $2^{-\Delta \Delta \mathrm{Ct}}$ method.

\section{Bioinformatics analysis}


Related miRNAs and their pathways were analyzed using Funrich software. The regulatory relationships between miRNAs and target genes were determined using TargetScan (http://www.targetscan.org/). Selected miRNAs were validated in hypoxia induced EVs that were secreted by HK2 cells.

\section{Luciferase reporter assay}

The NLRC5 3'UTR (3' untranslated region) luciferase reporter plasmid was created by ligation of the miR-125b-5p binding motif of NLRC5 3'UTR into the pmirGLO Dualluciferase miRNA target expression vector (Promega, Madison, WI, USA). Empty luciferase reporter plasmid was used as a control. Cells were seeded into 96-well plates. HK2 cells were co-transfected with pmir-GLO-NLRC5 or pmir-GLO empty vector using the Lipofectamine2000 transfection reagent (Thermo Fisher Scientific). After incubation with miR-125b-5p or control for $48 \mathrm{~h}$, luciferase activities of firefly and Renilla were measured in a DualLuciferase Reporter Assay System (Promega, Madison, WI, USA) according to the manufacturer's instructions.

\section{Periodic acid-Schiff staining}

To detect renal tubule injury, kidneys were fixed with $4 \%$ paraformaldehyde, deparaffinized, hydrated, and then embedded in paraffin. Further, tissues were sectioned ( $4 \mu \mathrm{m})$ and stained with PAS using PAS staining kit (G1008, Servicebio, Wuhan, China) according to the manufacturer's instructions. Histopathological score was determined by an experienced pathologist who was blinded to the renal tubule injury status of the mice. Staining scores were based on the grading of tubular cell necrosis, swelling, tubular casts, brush border loss, and interstitial infiltration by multi-nucleated cells in 10 randomly selected non-overlapping fields (magnification, $200 \times$ ). According to the severity of changes on a semi-quantitative scale, staining was graded as follows: no injury $=0$, $\operatorname{mild}=1$, moderate $=2$, severe $=3$, and very severe $=4$.

\section{Serum creatinine assay}

Blood samples were obtained through cardiac puncture at indicated times. Further, samples 
were incubated at room temperature for $1 \mathrm{~h}$ and then, centrifuged at 3,000 rpm for $10 \mathrm{~min}$ at $4{ }^{\circ} \mathrm{C}$. The upper serum was separated and the creatinine level was measured in mouse serum using commercial creatinine assay kit (C011-2, NJJC-Bio, Nanjing, China) according to the manufacturer's instructions.

\section{Immunohistochemical staining}

Kidney tissue sections were fixed with paraformaldehyde, paraffin-embedded, and stained with an immunohistochemical kit (G1206, ServiceBio, Wuhan, China). Antigen was retrieved using citric acid ( $\mathrm{pH} 6.0$ ), and endogenous peroxidase was removed with $3 \% \mathrm{H}_{2} \mathrm{O}_{2}$. Sections were incubated overnight with primary antibody polyclonal rabbit anti-mouse NLRC5 (1:200, LS-B5328, LifeSpan) at $4{ }^{\circ} \mathrm{C}$. Sections were washed with PBS and then, incubated with HRP-conjugated Affinipure Goat anti-rabbit IgG for $30 \mathrm{~min}$, followed by DAB. Finally, samples were counterstained with hematoxylin. NLRC5 staining was assessed by two independent pathologists and calculated as sum of the intensity score and the positive staining cell score. Staining was graded based on the following criteria: no staining $=0-1$, weak staining $=2-3$, moderate staining $=4-5$, and strong staining $=6$.

\section{Statistical analysis}

All data have been expressed as mean \pm SD. Statistical analysis was conducted using the SPSS 17.0 statistical software and GraphPad 6.0. Experimental data were first assessed for normal distribution and homogeneity of variance. For data with a normal distribution and homogeneity of variance, two-group mean comparison was conducted by independentsample t-test. Multi-group means were compared using one-way ANOVA. Multi-group means at different time points were analyzed by two-way ANOVA. $\mathrm{P}<0.05$ was considered statistically significant. Each experiment was independently performed at least three times.

\section{Results}

\section{Characterization of HK2 cell-derived EVs}

This study used a series of experiments to identify EVs. TEM analysis showed that HK2 cellderived EVs were $30 \mathrm{~nm}$ to $300 \mathrm{~nm}$ in size, and resembled round or elliptical lipid bilayer 
vesicles (Fig. 1A). Western blot analysis of marker proteins revealed that EVs show positively express CD63, HSP70, and CD81 (Fig. 1B).

\section{Hypoxia induced HK2 cell-derived EVs alleviate renal I/R injury in mice}

To investigate the protective effect of hypoxia induced HK2 cell-derived EVs in I/R injury, mice were categorized into four groups: sham surgery (sham), bilateral renal I/R injury injected with PBS (IRI+PBS), EVs from HK2 cells cultured under normoxic conditions (IRI+NOR-EVs), and EVs from hypoxia induced HK2 cells (IRI+HYP-EVs).

Morphological changes were observed to be alleviated in EV-treated groups and showed a statistically significant difference compared to that observed in the IRI+PBS group. Compared to the IRI+NOR-EVs group, there was a significant improvement in morphological changes of IRI+HYP-EV group (Figs. 2A, 2B). Furthermore, renal function was evaluated by serum creatinine (Scr) levels. Results showed that compared to sham group, Scr levels were significantly upregulated in the IRI+PBS group. Moreover, Scr levels of the EVs groups were observed to be lower compared to that observed in IRI+PBS group. Notably, significant improvement in the renal function of IRI+HYP-EV-treated mice was observed (Fig. 2C).

\section{Certain EV-miRNAs significantly alter expression under hypoxia}

MiRNA sequencing analysis indicated that compared to normoxic group, expression of 16 EV-miRNAs that were isolated from hypoxia-induced HK2 cells, were found to be significantly upregulated. To verify miRNA sequencing data, we conducted RT-PCR assay. We found that compared to normoxic group, four hypoxia-induced HK2 cell-derived EVmiRNAs were significantly upregulated, which included miR-125b-5p $(\mathrm{P}=0.0236)$, miR-3305P ( $\mathrm{P}=0.0044)$, miR-219a-2-3p ( $\mathrm{P}=0.0006)$, and miR-127-3p ( $\mathrm{P}=0.0004)$ (Fig. 3).

\section{Bioinformatics analysis and search for target genes}

We analyzed the four upregulated EV-miRNAs using bioinformatic tools and found only miR-125b-5p and miR-330-5P were closely related to the PI3K-AKT pathway in renal I/R injury $(\mathrm{P}<0.05)$ (Figs. 4A, 4B). Furthermore, we screened the predicted target genes of the two EV-miRNAs and selected NLRC5 as it plays an important role in renal I/R injury via the PI3K-AKT pathway. In addition, it has been reported that downregulated expression or deletion of NLRC5 can prevent renal I/R injury[23]. Bioinformatics analysis revealed that 
miR-125b-5p had a binding site in the 3'-UTR of NLRC5. Accordingly, we investigated the relationship between miR-125b-5p and NLRC5 in subsequent studies (Figs. 4C, 4D).

\section{NLRC5 is targeted by miR-125b-5p in HK2 cells}

We transfected HK2 cells with the miR-125b-5 negative control (miR-NC) or miR-125b-5p mimics (miR-MI). RT-PCR analysis of HK2 cells and their secreted EVs revealed that miRNA-125b-5p expression in miR-MI-transfected HK2 cells was 7-fold higher than that observed in miR-NC-transfected HK2 cells $(\mathrm{P}=0.0054)$. Subsequently, we extracted isolated EVs that were secreted from miR-MI-transfected and miR-NC-transfected HK2 cells. RTPCR analysis showed that miRNA-125b-5p expression level in EVs of the miR-MI group was over 1,400-fold higher than that observed in EVs of the miR-NC group ( $\mathrm{P}=0.0001)$ (Fig. 5A). Further, we analyzed NLRC5 mRNA expression by RT-PCR that was extracted from miR-MI-transfected and miR-NC-transfected HK2 cells. We found that the mRNA of NLRC5 expression in miR-MI-transfected HK2 cells was significantly lower than that observed in miR-NC-transfected HK2 cells $(\mathrm{P}=0.0002)$ (Fig. 5B). Furthermore, NLRC5 expression level in miR-MI-transfected HK2 cells was observed to be significantly lower than that expressed in miR-NC-transfected HK2 cells (Fig. 5C). Subsequently, luciferase reporter assay showed that miR-MI significantly reduced the luciferase activity on pmir-GLO-NLRC5 transfection, whereas NC had no significant effect on the luciferase activity on pmir-GLO-NLRC5 transfection. Similarly, miR-MI and miR-NC had no significant effect on the luciferase activity on transfecting the pmir-GLO empty vector. Thus, results indicated that miR-125b-5p specifically targeted NLRC5 ( $\mathrm{P}=0.028)$ (Fig. 5D).

\section{EVs containing miRNA-125b-5p reduced renal I/R injury by targeting NLRC5}

To study the protective effect of miRNA-125b-5p on renal I/R injury by targeting NLRC5, we categorized mice into four groups: sham group, IRI+PBS group, EVs from HK2 cells transfected with miR-NC (IRI+NC-EVs), and EVs from HK2 cells transfected with miR-MI (IRI+MI-EVs).

Compared to sham group, all renal tubules in the $\mathrm{I} / \mathrm{R}$ injury groups were found to be severely injured. The degree of renal tubule injury was found to be significantly alleviated on treating with EVs. Compared to IRI+NC-EVs group, the degree of renal tubule morphological injury showed a significant improvement in the IRI+MI-EV group (Figs. 6A, 6B). Scr levels in the sham group was found within normal limits. Compared to sham group, Scr levels were found 
to be significantly upregulated in the IRI+PBS group. Subsequently, compared to the IRI+PBS group, the Scr levels of the EV groups were found to be lower. Moreover, IRI+MIEVs group were observed to exhibit remarkable alleviation in renal injury (Fig. 6C). NLRC5 expression was immunohistochemically assessed in the paraffin sections of mouse kidney tissues. Compared to the sham group, NLRC5 expression levels in the kidney tissues of mice with renal I/R injury were found to be significantly upregulated. Moreover, NLRC5 was found to be mainly expressed in the renal tubule region. Compared to the IRI+PBS group, NLRC5 expression level in the IRI+MI-EVs group was found to be significantly downregulated (Fig. 6D).

\section{Discussion}

In our previous study, we first established a rat model of RIPC such that the left kidney was protected from I/R injury by the right kidney IPC. We found that hypoxia induced renal tubule epithelial cells release functional EVs that mediated renal I/R injury remission[24]. Tuorkey et al.[25] reported a similar renal RIPC model and demonstrated that kidney RIPC shows exceptional anti-I/R injury efficacy.

Release and uptake of EVs play a key role in the disease progression of kidney[24, 25]. In our previous and present studies, we have found that EVs from hypoxia preconditioned HK2 cells alleviate renal I/R injury, which might account for RIPC.However, the mechanism by which EVs exhibit protective effect against renal I/R injury remains unclear. EVs have been considered to function by releasing their cargoes into receptor cells[26]. miRNAs are one of the most important cargoes in EVs, which have been suggested to play an important role in renal I/R injury, and has attracted immense interest from researchers[13-16]. Kaucsar et al.[27] reported that expression of miR-21, miR-17-5p, and miR-106a was significantly elevated during the maintenance and recovery phase of renal I/R injury-induced AKI. In addition, the correlation between miR-21 and miR-17-5p has been shown to be affected by the renal reperfusion phase. Furthermore, Aguado et al.[28] has described the novel role of miR-127 in cell adhesion and regulating hypoxia-inducible factor-1 $\alpha$ (HIF-1 $\alpha)$ expression. They found that miR-127 and kinesin family member 3B (KIF3 $\beta$ ) could be used as key mediators in regulating renal I/R injury; thereby, treating ischemic injury caused to the kidneys. Moreover, we have also observed that the mesenchymal stromal cells derived EVs protect renal I/R injury by transporting miRNAs[29, 30]. Thus, it is suggested that miRNA 
enclosed in EVs of hypoxia preconditioned HK2 cells may play an important role in renal I/R injury.

In the present study, we extracted the HK2 cell-derived EVs by ultra-high speed centrifugation and performed characterization analysis. After injecting normoxic-induced and hypoxia-induced HK2 cell-derived EVs in mice, we found that EVs from hypoxia pretreated HK2 cells had enhanced therapeutic effects, which was consistent with our previous findings[10, 22]. Further, we analyzed the differentially expressed HK2 cell-derived EVmiRNAs under two circumstances by miRNA sequencing, and verified the expression of 16 EV-miRNAs by RT-PCR. Results confirmed that four EV-miRNAs were elevated under hypoxic conditions. Bioinformatics analysis was performed on these elevated miRNAs, and only miR-125b-5p and miR-330-5P were observed to be highly enriched in the PI3K-AKT pathway. Significance of the PI3K-AKT pathway in renal I/R injury has been extensively reported[31, 32]. After screening all the predicted target genes, we focused on NLRC5, a potential target gene of miR-125b-5p. Thus, we hypothesized that miR-125b-5p might have specifically targeted NLRC5 and subsequently, attenuated renal I/R inhury.

In contrast, we transfected HK2 cells with miR-MI and miR-NC, and determined miR-125b$5 p$ expression by RT-PCR assay. Results revealed that miRNA-125b-5p significantly upregulated in HK2 cells when transfected with miR-MI compared to miR-125b-5p expression in miR-NC-transfected HK2 cells. Interestingly, abundant expression of miRNA$125 \mathrm{~b}-5 \mathrm{p}$ was found to be remarkably significant in EVs than in HK2 cells. Thus, our results demonstrated that EV-associated miR-125b-5p might have played a significant role in renal protection.

NLRC5 is the largest member of in the NOD-like receptors (NLR) family, which has a typical NLR structure. Furthermore, NLRC5 is an important regulator of the inflammasome signaling pathways, type I interferons, and major histocompatibility complex I[33]. NLRC5 knockdown has been reported to significantly improve renal I/R injury[23, 34]. We found that both of mRNA and protein expression levels of NLRC5 was significantly downregulated in miR-125b-5p overexpression HK2 cells. It is known from the database that miR-125b-5p has a binding site on 3'-UTR of NLRC5. Notably, luciferase reporter assay confirmed that miRNA-125b-5p could directly bind to NLRC5.

For animal study, EVs from miR-MI-transfected or miR-NC-transfected were injected in 
mice. Results showed that increased expression of miRNA-125b-5p in EVs was found to significantly reduce the degree of renal tubule morphological injury and also improve renal function. Moreover, from immunohistochemical analysis, we found that EVs from the miRMI-transfected HK2 cells suppressed the NLRC5 expression in renal tissues with I/R injury. Thus, it proved that the important role of miRNA-125b-5p/NLRC5 biological axis in alleviating renal I/R injury by EVs from hypoxia pretreated HK2 cells.

\section{Conclusion}

In summary, this study investigated the alterations in hypoxia induced EV-miRNAs, and explored the regulatory molecules targeted by miRNA-125b-5p by bioinformatics analysis. MiRNA-125b-5p contained in EVs from hypoxia pretreated HK2 cells was found to exert protective effects on mouse renal I/R injury mainly by inhibiting NLRC5. This may be a potential mechanism involved in the protection effects of remote ischemia precondition for renal I/R.

\section{Conflicts of interest}

The authors declare that they have no potential conflicts of interest to disclose.

\section{Author contruibutions}

Conceived and designed the experiments: GYZ, MC and SQC. Performed the experiments: KX, LZ, LZ, SWQ, SS, and XYZ. Analyzed the data: GYZ,BX,LZ and LFZ. Wrote the paper: GYZ,KX and ZZ. Funding support: GYZ and XYZ. All authors read and approved the final manuscript.

\section{Funding}

This work was supported by the National Nature Science Foundation of China (No. 81670632 and No.81900618); Natural Science Foundation of Jiangsu Province of China (SBK2020042417).

\section{References}

1. Andreucci M, Faga T, Pisani A, Perticone M, Michael A. The ischemic/nephrotoxic acute kidney injury and the use of renal biomarkers in clinical practice. Eur J Intern Med. 2017; 
39: $1-8$.

2. Salvadori M, Rosso G, Bertoni E. Update on ischemia-reperfusion injury in kidney transplantation: Pathogenesis and treatment. World J Transplant. 2015; 5: 52-67.

3. Rosner MH, Okusa MD. Acute kidney injury associated with cardiac surgery. Clinical journal of the American Society of Nephrology : CJASN. 2006; 1: 19-32.

4. Sedaghat Z, Kadkhodaee M, Seifi B, Salehi E. Inducible and endothelial nitric oxide synthase distribution and expression with hind limb per-conditioning of the rat kidney. Arch Med Sci. 2019; 15: 1081-91.

5. Kharbanda RK, Nielsen TT, Redington AN. Translation of remote ischaemic preconditioning into clinical practice. Lancet. 2009; 374: 1557-65.

6. Davidson SM, Yellon DM. Exosomes and cardioprotection - A critical analysis. Mol Aspects Med. 2018; 60: 104-14.

7. Giricz Z, Varga ZV, Baranyai T, Sipos P, Paloczi K, Kittel A, et al. Cardioprotection by remote ischemic preconditioning of the rat heart is mediated by extracellular vesicles. J Mol Cell Cardiol. 2014; 68: 75-8.

8. Pan T, Jia P, Chen N, Fang Y, Liang Y, Guo M, et al. Delayed Remote Ischemic Preconditioning ConfersRenoprotection against Septic Acute Kidney Injury via Exosomal miR21. Theranostics. 2019; 9: 405-23.

9. Ma H, Xu Y, Zhang R, Guo B, Zhang S, Zhang X. Differential expression study of circular RNAs in exosomes from serum and urine in patients with idiopathic membranous nephropathy. Arch Med Sci. 2019; 15: 738-53.

10. Zhang G, Yang Y, Huang Y, Zhang L, Ling Z, Zhu Y, et al. Hypoxia-induced extracellular vesicles mediate protection of remote ischemic preconditioning for renal ischemiareperfusion injury. Biomedicine \& Pharmacotherapy. 2017; 90: 473-8.

11. Colombo M, Raposo G, Thery C. Biogenesis, secretion, and intercellular interactions of exosomes and other extracellular vesicles. Annu Rev Cell Dev Biol. 2014; 30: 255-89.

12. Pan BT, Johnstone RM. Fate of the Transferrin Receptor during Maturation of Sheep Reticulocytes Invitro - Selective Externalization of the Receptor. Ce11. 1983; 33: 967-77.

13. Li M, Zeringer E, Barta T, Schageman J, Cheng A, Vlassov AV. Analysis of the RNA content of the exosomes derived from blood serum and urine and its potential as biomarkers. Philos Trans R Soc Lond B Biol Sci. 2014; 369.

14. Alves LR, Peres da Silva R, Sanchez DA, Zamith-Miranda D, Rodrigues ML, Goldenberg S, et al. Extracellular Vesicle-Mediated RNA Release in Histoplasma capsulatum. mSphere. 2019; 4.

15. Liu T, Zhang Q, Zhang J, Li C, Miao YR, Lei Q, et al. EVmiRNA: a database of miRNA profiling in extracellular vesicles. Nucleic Acids Res. 2019; 47: D89-D93.

16. Qiu G, Zheng G, Ge M, Wang J, Huang R, Shu Q, et al. Mesenchymal stem cel1-derived extracellular vesicles affect disease outcomes via transfer of microRNAs. Stem Cell Res Ther. 2018; 9: 320.

17. Wei QQ, Liu Y, Liu PY, Hao JL, Liang MY, Mi QS, et al. MicroRNA-489 Induction by HypoxiaInducible Factor-1 Protects against Ischemic Kidney Injury. Journal of the American Society of Nephrology. 2016; 27: 2784-96.

18. Keasey MP, Scott HL, Bantounas I, Uney JB, Kelly S. MiR-132 Is Upregulated by Ischemic 
Preconditioning of Cultured Hippocampal Neurons and Protects them from Subsequent OGD Toxicity. J Mol Neurosci. 2016; 59: 404-10.

19. Ren GL, Zhu J, Li J, Meng XM. Noncoding RNAs in acute kidney injury. Journal of Cellular Physiology. 2019; 234: 2266-76.

20. Xu X, Kriegel AJ, Liu Y, Usa K, Mladinov D, Liu H, et al. Delayed ischemic preconditioning contributes to renal protection by upregulation of miR-21. Kidney international. 2012; 82: $1167-75$.

21. Wei Q, Liu Y, Liu P, Hao J, Liang M, Mi QS, et al. MicroRNA-489 Induction by HypoxiaInducible Factor-1 Protects against Ischemic Kidney Injury. J Am Soc Nephrol. 2016; 27: 2784-96.

22. Zhang L, Liu H, Xu K, Ling Z, Huang Y, Hu Q, et al. Hypoxia preconditioned renal tubular epithelial cell-derived extracellular vesicles alleviate renal ischaemia-reperfusion injury mediated by the HIF-1alpha/Rab22 pathway and potentially affected by microRNAs. International journal of biological sciences. 2019; 15: 1161-76.

23. Li Q, Wang Z, Zhang Y, Zhu J, Li L, Wang X, et al. NLRC5 deficiency protects against acute kidney injury in mice by mediating carcinoembryonic antigen-related cell adhesion molecule 1 signaling. Kidney international. 2018; 94: 551-66.

24. Zhang G, Yang Y, Huang Y, Zhang L, Ling Z, Zhu Y, et al. Hypoxia-induced extracellular vesicles mediate protection of remote ischemic preconditioning for renal ischemiareperfusion injury. Biomed Pharmacother. 2017; 90: 473-8.

25. Tuorkey MJ. Kidney remote ischemic preconditioning as a novel strategy to explore the accurate protective mechanisms underlying remote ischemic preconditioning. Interv Med Appl Sci. 2017; 9: 20-6.

26. Zhang ZG, Chopp M. Exosomes in stroke pathogenesis and therapy. J Clin Invest. 2016; 126: $1190-7$.

27. Kaucsar T, Revesz C, Godo M, Krenacs T, Albert M, Szalay CI, et al. Activation of the miR-17 Family and miR-21 During Murine Kidney Ischemia-Reperfusion Injury. Nucleic Acid Ther. 2013; 23: 344-54.

28. Aguado-Fraile E, Ramos E, Saenz-Morales D, Conde E, Blanco-Sanchez I, Stamatakis K, et al. miR-127 Protects Proximal Tubule Cells against Ischemia/Reperfusion: Identification of Kinesin Family Member 3B as miR-127 Target. Plos One. 2012; 7.

29. Zou XY, Yu Y, Lin S, Zhong L, Sun J, Zhang G, et al. Comprehensive miRnA Analysis of Human Umbilical Cord-Derived Mesenchymal Stromal Cells and Extracellular Vesicles. Kidney \& blood pressure research. 2018; 43: 152-61.

30. Zou X, Zhang G, Cheng Z, Yin D, Du T, Ju G, et al. Microvesicles derived from human Wharton's Jelly mesenchymal stromal cells ameliorate renal ischemia-reperfusion injury in rats by suppressing CX3CL1. Stem cell research \& therapy. 2014; 5: 40.

31. Zhang GS, Wang QL, Zhou Q, Wang RJ, Xu MZ, Wang HP, et al. Protective Effect of Tempol on Acute Kidney Injury Through PI3K/Akt/Nrf2 Signaling Pathway. Kidney Blood Press R. 2016; 41: 129-38.

32. Hu S, Zhang YX, Zhang M, Guo YC, Yang P, Zhang S, et al. Aloperine Protects Mice against Ischemia-Reperfusion (IR)-Induced Renal Injury by Regulating PI3K/AKT/mTOR Signaling and AP-1 Activity. Mo1 Med. 2015; 21: 912-23. 
33. Kobayashi KS, van den Elsen PJ. NLRC5: a key regulator of MHC class I-dependent immune responses. Nat Rev Immunol. 2012; 12: 813-20.

34. Wang C, Tang Y, Yang D, Huang Y. NLRC5, a novel role in cardiac fibrosis. Biomed Pharmacother. 2017; 96: 1567-8. 
A

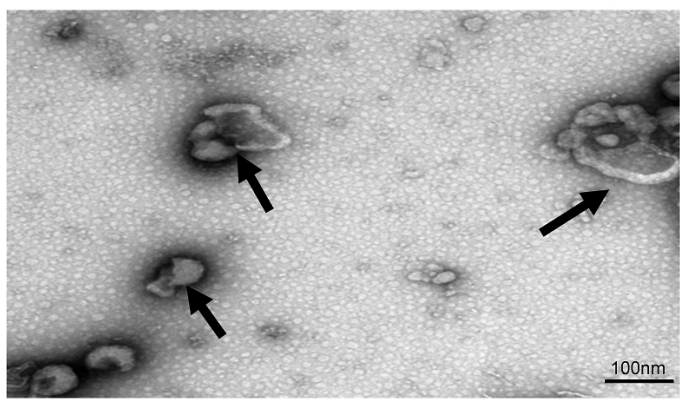

C

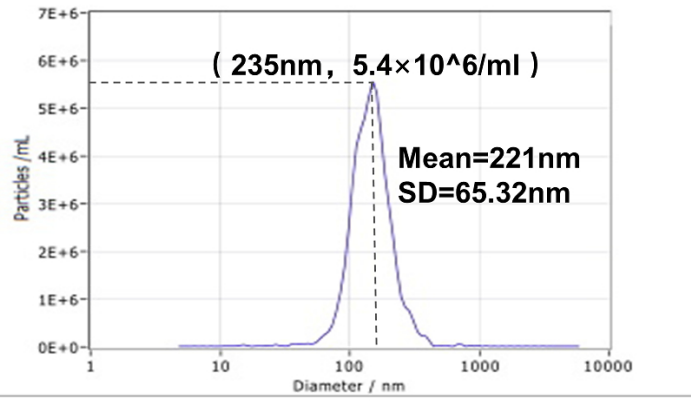

B

KDa

CD63

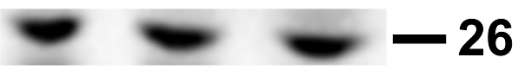

HSP70

$-70$

CD81

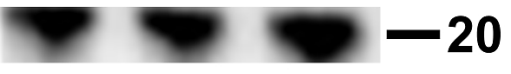

D

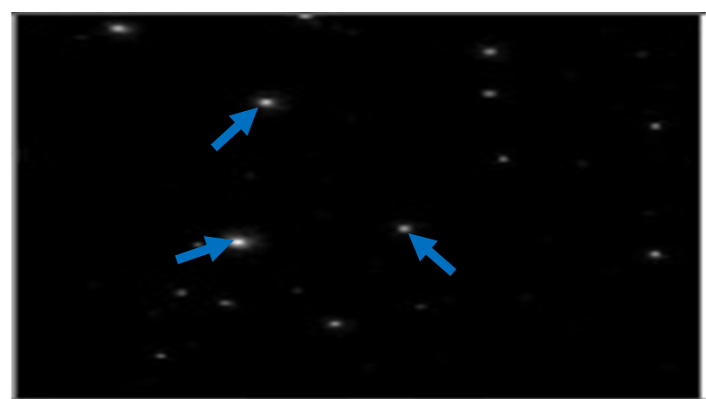

Figure 1. (A) TEM assay for HK2 derived EVs. (B) Surface marker (CD81, CD63, and HSP70) of EVs from HK2 cells. (C) NTA assay for EVs. (D) Microscopy of the molecular Brownian motion of EVs in NTA. 
A
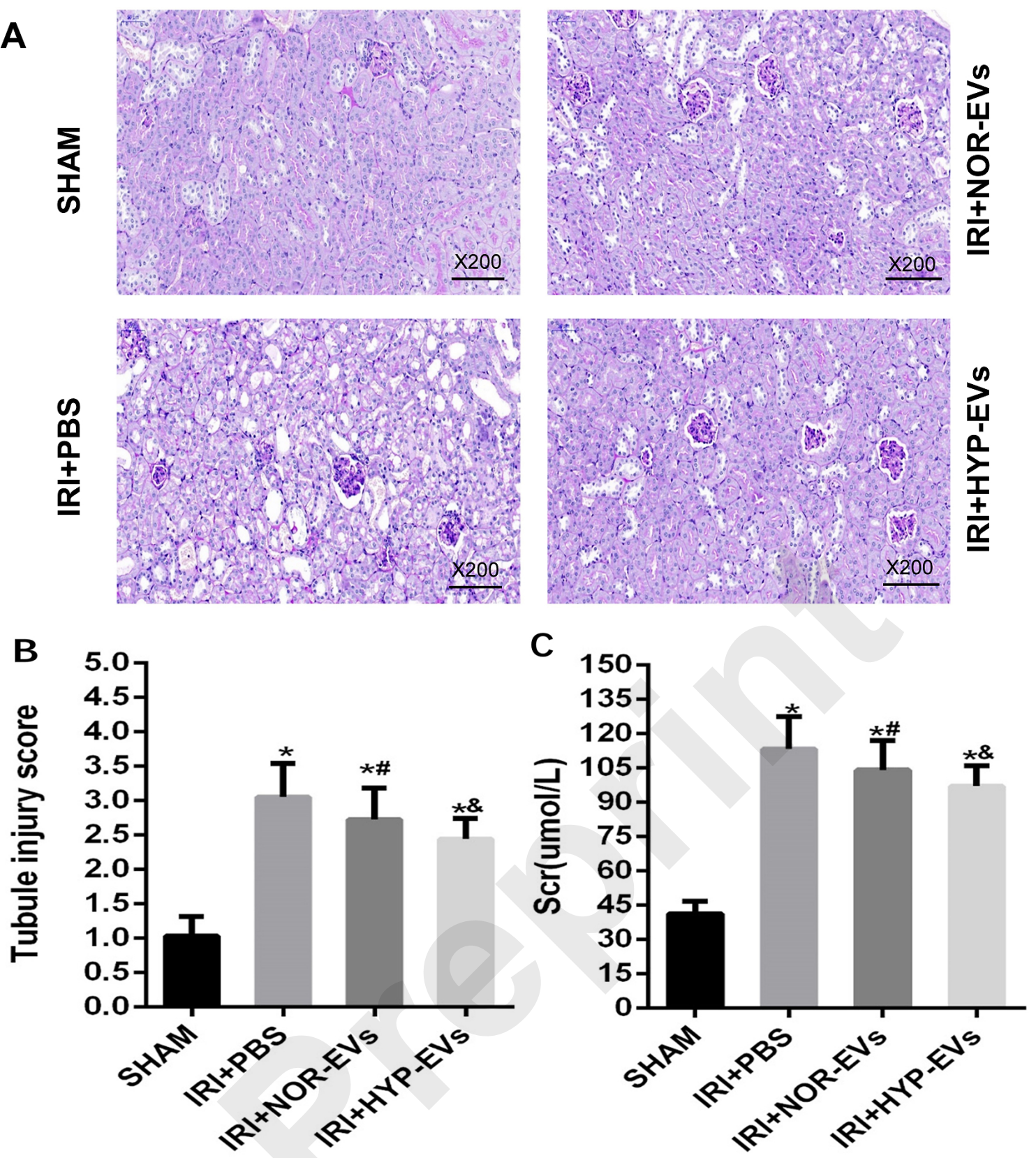

Figure 2. (A) Representative renal sections from different groups (PAS staining, magnification 200x); (B) Renal tubule injury scores for each group. It was showed that hypoxia induced HK2 cell-derived EVs reduced renal tubule injury caused by renal I/R. (C) Serum creatinine levels for different groups. (Groups: sham surgery (SHAM), renal ischaemia reperfusion injected with PBS (IR+PBS), with EVs from HK2 cells cultured under normoxic conditions (IR+NOR-EVs) or with EVs from hypoxia pretreated HK2 cells (IR+HYPEVs) ${ }^{*} \mathrm{P}<0.05$ compared with SHAM group; \#P < 0.05 compared with IRI+PBS group; \&P $<0.05$ compared with IRI+NOR-EVs groups). 


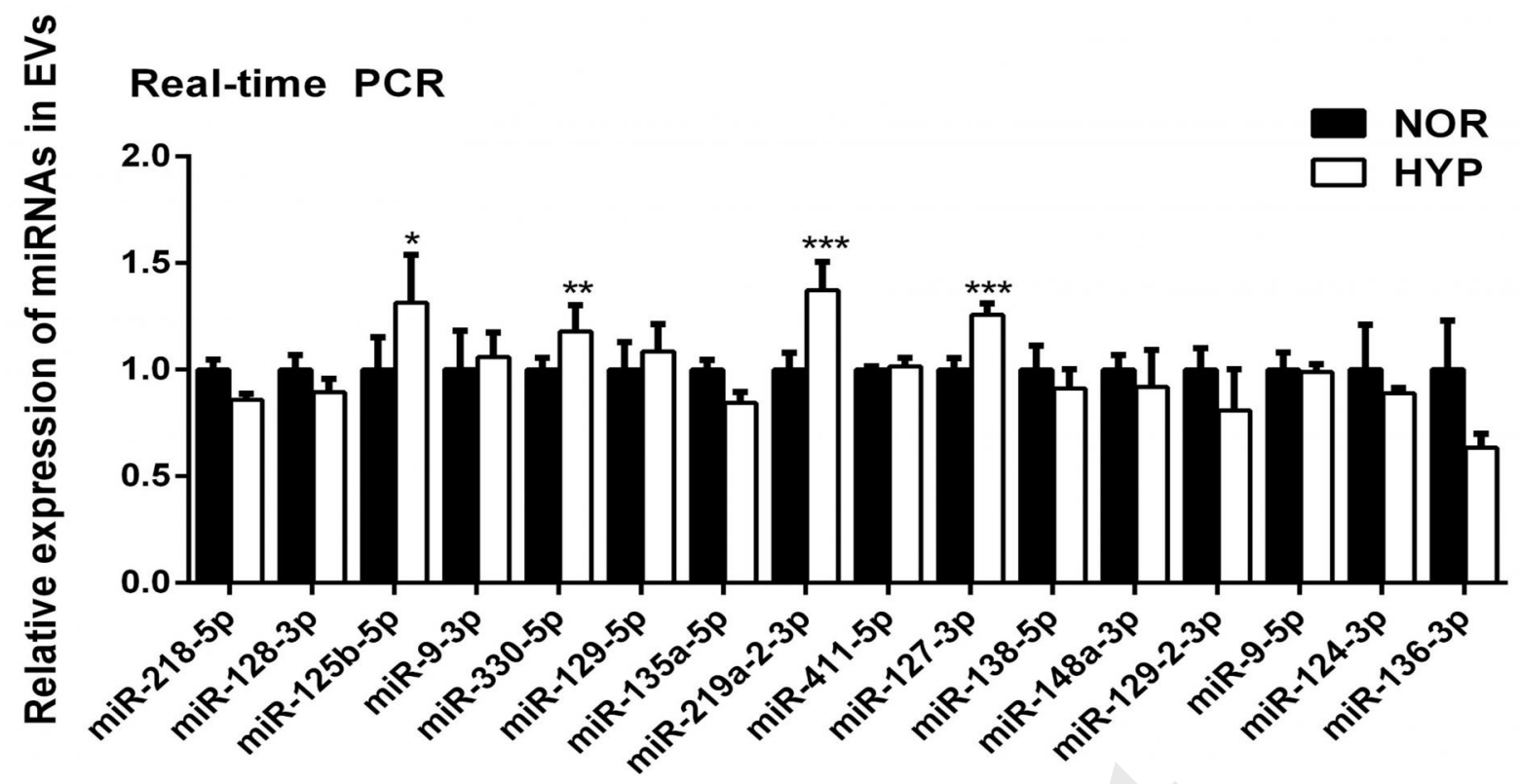

Figure 3. RT-PCR results for 16 candidate miRNAs which was found changed by sequencing analysis. ( $\left.{ }^{*} \mathrm{P}<0.05,{ }^{* *} \mathrm{P}<0.01,{ }^{* *} \mathrm{P}<0.001\right)$. 

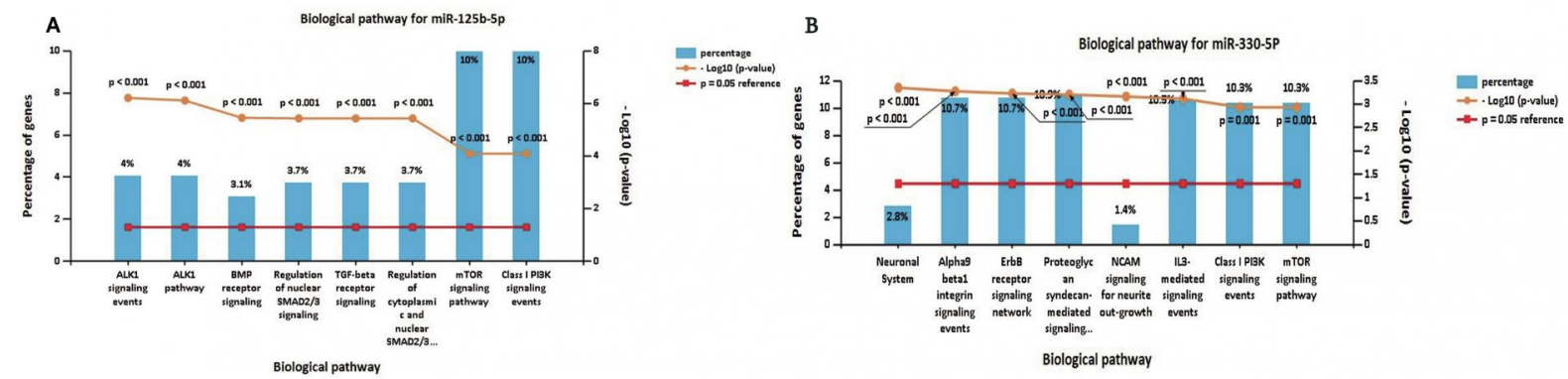

Human NLRC5 ENST00000436936.1 3' UTR length: 2005

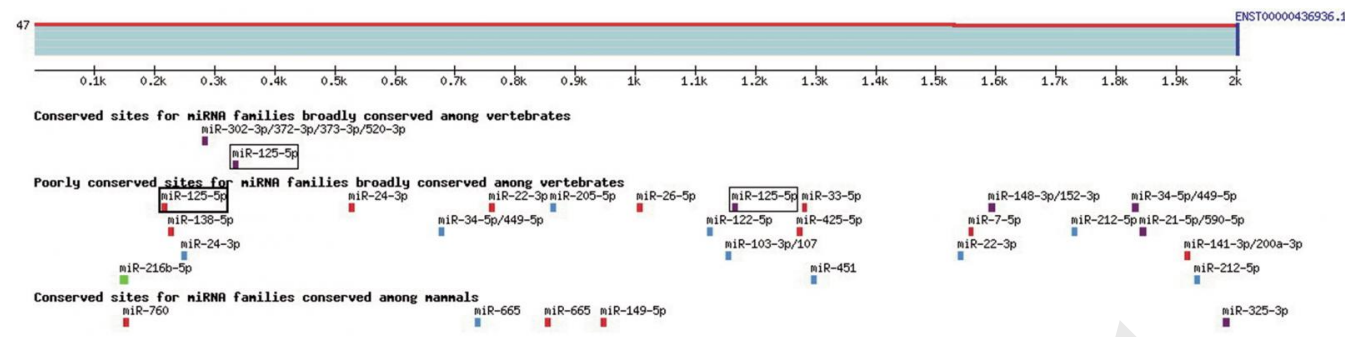

D

Position 331-338 of NLRC5 3' UTR

hsa-miR-125b-5p

5'...CAggaAgAuAgaccucucaggGa...

I I I I i i

3' ...AGUGUUCAAUCCCAĠÁĠÚĊĊU...

Figure 4. (A, B) Pathway analysis showed that miR-125b-5p and miR-330-5P were highly enriched in the PI3K-AKT pathway. (C, D) Bioinformatics analysis revealed that the $3^{\prime} U T R$ of NLRC5 has binding sites for miR-125b-5p. 
A

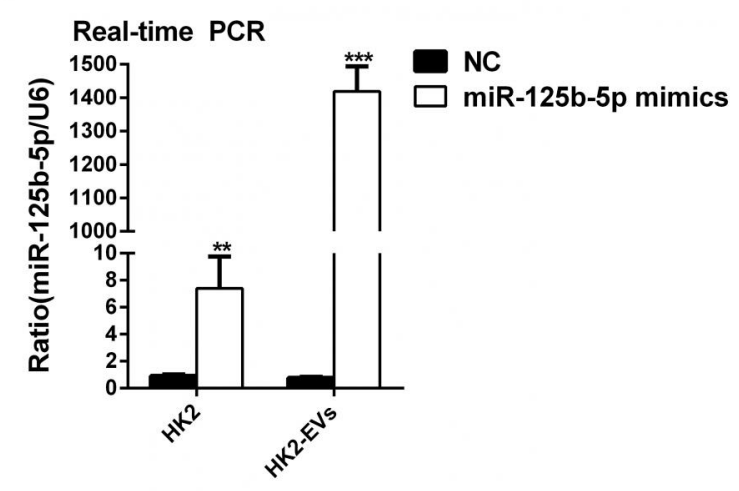

C

Western blot of NLRC5

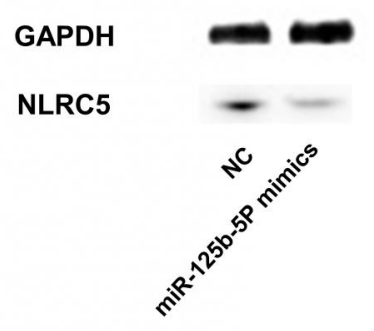

B

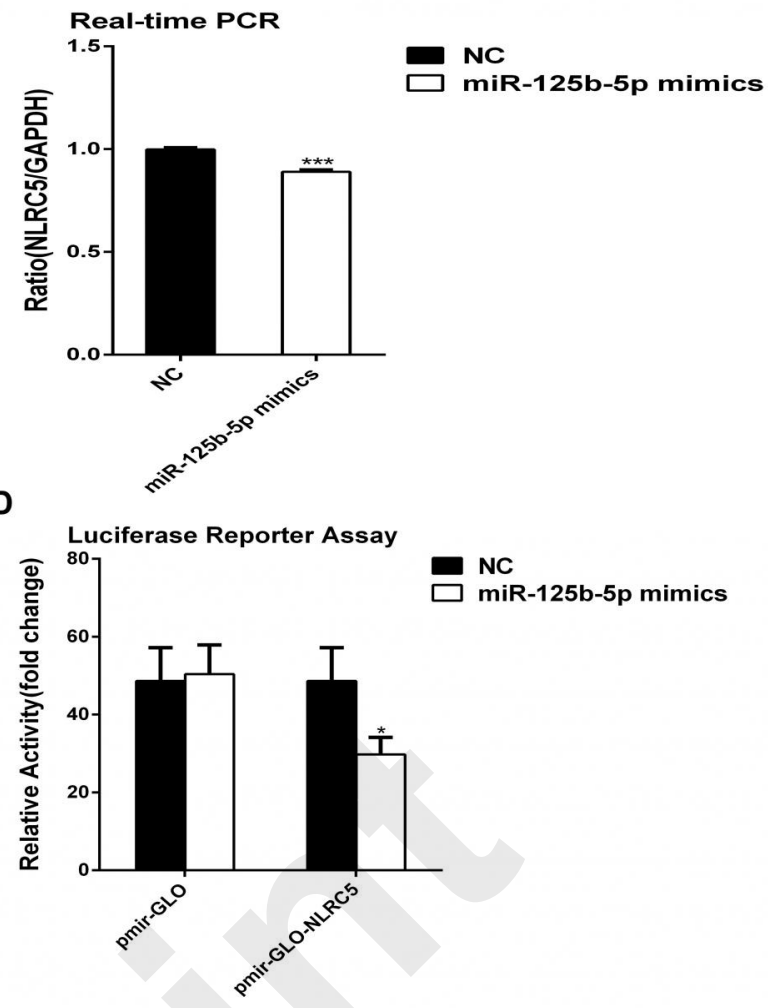

Figure 5. (A) miRNA-125b-5p expression increased in HK2 cells transfected with miR-125b-5p mimics (miR-Ml) and in their secreted EVs. (B) The mRNA of NLRC5 decreased significantly in HK2 cells transfected with miR-MI. (C) The expression of NLRC5 protein significantly decreased in HK2 cells transfected with miR-MI. (D) Luciferase reporter assay showed that miRNA-125b-5p directly bind to NLRC5 (NC, miR-125b-5p negative control group. pmir-GLO, empty GLO vector; pmir-GLO-NLRC5, GLO vector with NLRC5 biding site; ${ }^{*} \mathrm{P}<0.05$, ${ }^{* *} \mathrm{P}<0.01,{ }^{* * *} \mathrm{P}<0.001$, vs NC group; \# $\mathrm{P}<0.05$, vs pmir-GLO with miR-125-5p mimics). 

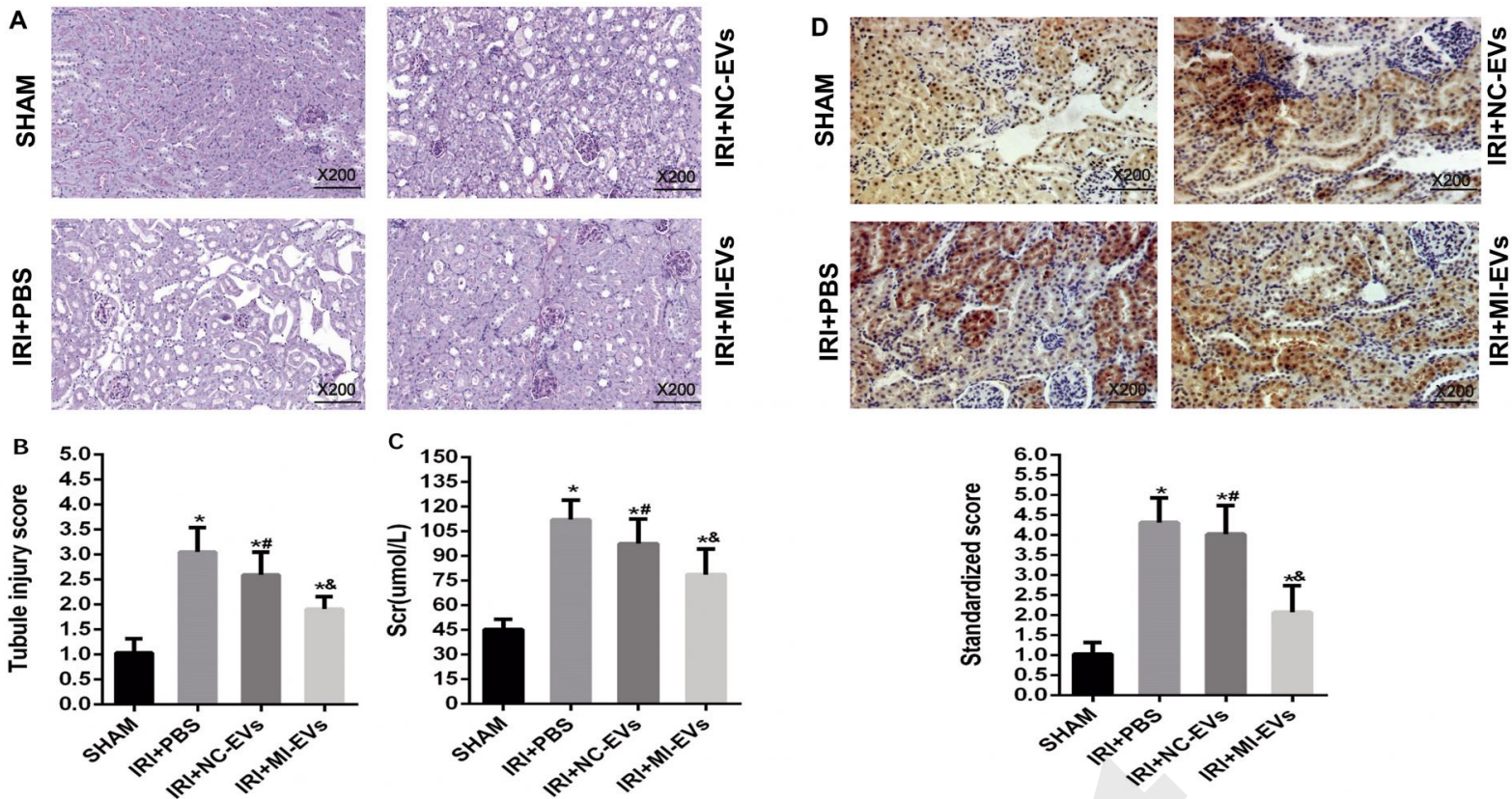

Figure 6. (A) Representative renal sections from different groups (PAS staining, magnification 200x);. (B) Renal tubule injury scores and statistical analyses of each group. (C) Serum creatinine levels for different groups. (D) Immunohistochemistry assay for NLRC5 in paraffin sections of kidney tissues of each group (magnification 200x) and semiquantitive scoring for each group, (sham surgery (SHAM), renal ischaemia reperfusion injected with PBS (IR+PBS), with EVs from HK2 cells transfected with miR-125b-5p negative control (IR+NC-EVs) or with EVs from HK2 cells transfected with miR-125b-5p mimics(IR+HYP-EVs) ${ }^{*} \mathrm{P}<0.05$ vs SHAM group; \#P $<0.05$ vs IRI+PBS group; \&P $<0.05$ vs IRI+NC-EVs group). 